\title{
VIRTUALIZATION TECHNICAL THINKING WITHIN THE INFORMATION TECHNOLOGY
}

\author{
Robert Lis ${ }^{1}$ \\ 1 Department of Teaching Methods and Strategies, Lublin University of Technology, Nadbystrzycka 38, 20-618 \\ Lublin, Poland, e-mail: r.lis@pollub.pl
}

Received: 2014.09.25

Accepted: 2014.10.25

Published: 2014.12.01

\begin{abstract}
The article presents the possibilities of virtualization technical thinking within the information technology. This question expresses the need for virtualization of existing information systems to improve the conduct of business by the company. In resulting virtualization of the technical-computer thought on against existing needs of the functioning of 16-bit systems in 64-bit systems. Enables cost-effective use of excess capacity existing computing resources.
\end{abstract}

Keywords: virtualization, thinking technical-information.

\section{INTRODUKCTION}

The technical thinking consists in competent applying in intellectual activities both practical notions and judgements describing and clarifying the technique. To concern it can of different fields of technology. Nowadays almost an information technology is playing an important role in every field of technology and the human activity. She is developing at full speed. Is one of manifestations of this development virtualization of servers.

She evolved within a few last years from the technology being born to the seen function IT. Companies more and more often start using from her power to meet the changing business needs. Through virtualization of one's burdens, organizations can control and reduce costs, improving the scalability, the flexibility and the reach of computer systems simultaneously. Arising progress more and more is making aware, that virtualization alone in himself doesn't let companies build and perform business tasks. Denouement Hyper-V was first introduced as part of the system Windows Server 2008, and then extended and strengthened in the system Windows 2012. Hyper-V provides equipment investments of the server for business organizations of the tool for the operational research through the consolidation of many functions of the server in the form of separate virtual machines started on the single machine of the physical host. It is possible also to use Hyper-V in order effectively to act on many operating systems, in it operating systems different from Windows, so like for example altogether DOS on one physical server, and also to exploit the 64-bit power of processors.

The purpose of this article is to signal the need to find an optimal solution to streamline the running of the business in its current form. The use of proven, high-performance 16-bit ERP applications running under the DOS operating system becomes more difficult due to technological advances and the lack of compatibility ,,down“, current solutions. ERP software, particularly in a version for DOS systems have very specific requirements. It is eg. on the number of simultaneously open files.

DOS was able to open simultaneously to 255 files under the condition of proper configuring.

Parameter ,files" required for the accomplishment it is possible to configure in the different way only on 32-bit systems. Rates offered at present of the proportion of the screen are an additional obstacle. In this case a resolution should be a standard in the 4:3 format. This 
$640 \times 480$ of pixels is most often, more rarely $800 \times 600$ of pixels. Such a resolution should be kept for right proportions of letters and the arrangement of windows. Direct reference to data saved onto the disk being a consequence of the application among others ,,only" $640 \mathrm{~KB}$ of the main memory in current systems very much was modernised by initial versions DOS. Applying SSD disks which can work with the speed will be a good example of memory towards creating the buffer called the RAM, RAMdrive" in the destination of precipitating performing the operation you on very free at that time disks plate.

Every system of the ERP class performs operations, of which many times a document printout is a result. For a few years we have an already legally normalised possibility of the transmission of the documents for contracting parties without the signature and in an electronic form. However for the purposes of keeping records for the accountant and the possible tax audit documents having a force of law are most often printed on paper. Also tax documentation and less and less often tax declarations find their reflection in physics printouts. ERP systems designed for different sized companies usually implement sending codes to print directly to the printer via the parallel port. Unfortunately, the port has been almost entirely supplanted by USB, and multiple machines is no longer present. In addition, the matter is complicated by the fact that the use of heavy duty dot matrix printers have only LPT port. Operatings cost of these devices are limited to an exchange printing tapes every a few months or even years in case of occasional prints. Perhaps they aren't too fast, perhaps are too loud but the fact that they are able simultaneously to print a few copies of the same printout is also their advantage. For this reason users don't want to give these devices up.

Should also mention fiscal printouts which are a requirement of the completion of retail trades. Devices being used for it are communicating through a serial port. Even if a bought new

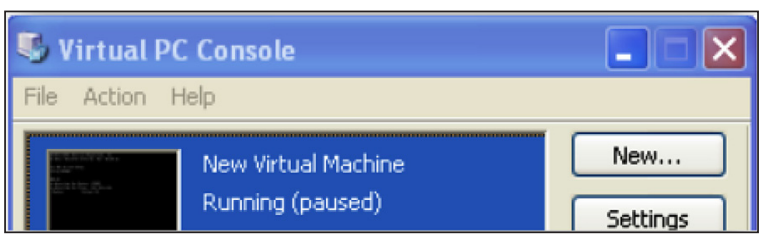

Fig. 1. Virtual machine in application Virtual PC fiscal printer having a universal USB port will stay it still signals on this port will be emulating serial communication. Can see it in the system after installed drivers which are always visible as ,high" COMM ports. it is necessary then to pay attention to the need for the change of the port number on one from acceptable through the ERP systems.

\section{USE OF 16-BIT APPLICATIONS ERP}

Some attempt to address the need for the use of ERP applications for DOS made Microsoft releasing system windows 7 in version 64-bit with additional software. Wanted to be able to work for applications that previously worked in the systems 16 -bit to 32-bit. In order to keep the compatibility he suggested a solution named VirtualPC. was it is an ovule and simultaneously confession of the producer to the fact that he exists work opportunities need the behaviour with older applications. VirtualPC was a simple concept of creating operating systems of guest "'in the system, of host. The solution lived to see to become topical to version 2007, in which flat Aka a possibility was blocked virtualization of system windows 98 . With next answer, revolutionary on account of the use free of charge, is HyperV. On purpose omitted dissolutions of other companies will stay for economic reasons.

Saying about virtualization as the indispensable tool enabling the service of ERP systems under DOS one should clarify this notion. On account of a lot of accessible definitions, I will use classifying solutions in terms of the running speed. With base wirtualizacji there were emula-

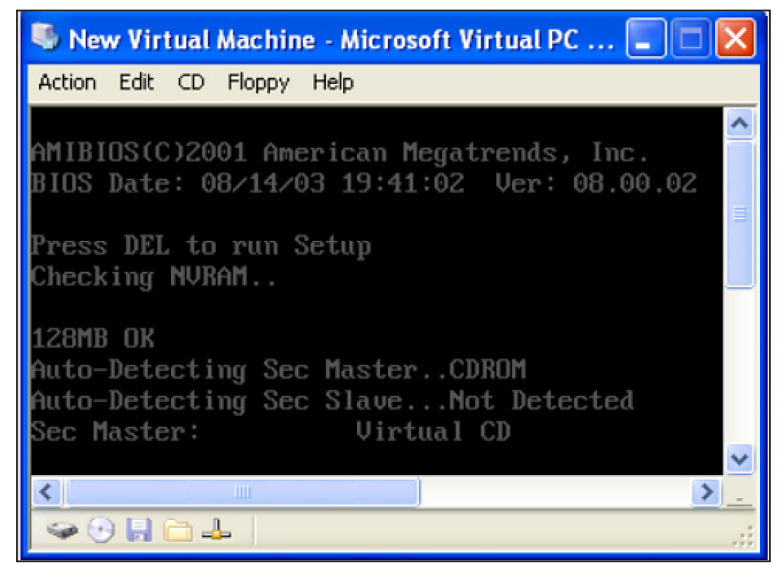

Fig. 2. Boot of the guest in the application Virtual PC 
tors of operating systems which thanks enable applying interpreting the Instruction work of applications written to other system than installed in the computer. I will use the name here moderators because main games written under Atari, Commodore and many other computers but the PC started on the platform were their beneficiary. Their manner of the work caused the toplevel emulation of the system of the guest what resulted up to 500 times in slower action than the system of the host. Next in virtualization Hypervisor is starting the special sequence of the VMXON instruction in the processor. In the native version virtualization in particular hypervisor - administrator virtualization, more efficient than the traditional emulation, the system of the guest is starting the host directly on the equipment this way, so that they as the most instruction make directly both he controls the equipment and is managing guest operating systems. Is maximizing in this way using the real equipment and providing software - machine virtualization only dangerous instructions. This model is a classic implementation of virtual machine architecture ; Primary hypervisors were used for testing. Modern equivalent enServer Citrix , VMware ESX / ESXi and Microsoft Hyper-V hypervisor.

The virtualization, in which hypervisors are in the traditional environment of the operating system and being on the next layer on the lower level of preference start the guest OS and hypervisor software running on the hardware allocates resources for running virtual systems. KVM, VirtualBox and Virtual PC are examples of virtualization hypervisor. As a result, Hyper -V works almost as good as the original system. However, it emulates a USB or audio inputs. If they are then you need to connect to the virtual machine via Remote Desktop.

\section{USING THE EXCESS OF THE POWER IN CURRENT COMPUTERS}

64-Bit architecture is being met today almost in every individual. 64-Bit processors and operating systems suiting them are a standard. Processors are characterized not one and a few not to say with a dozen or so physics cores. They often have a technology which is doubling the number of cores to several dozen logical cores.

Very much a lot of the main memory is in contemporary computers on account of for her relatively minimum price for the GB. a development of the so-called technology is supporting it double of the memory i.e. the memory put in pairs on the motherboard. Limiting to $4 \mathrm{~GB}$ 32-bit systems were able to address which was also changed on $64 \mathrm{~TB}$ in versions 64-bit.

The technological progress didn't pass bulk storages. The parallel record on magnetic plates increased "the packing density" causing information constant increasing the capacity and the price cutting. The development of the concept of very fast electronic disks of SSD caused, that very much a price for the megabyte magnetic disks offer which had diminished.

However the technological progress in this case a bit went too far ordering the operator using pointing devices as the feather, the mouse or the touch screen. Even harnessing bars code reader which by design entering the assortment into the system was supposed to hasten, in many cases will fail an examination for instance from the account of the lack of the bar code on goods.

A few ways to use the excess of accessible sources exist in contemporary computer machines. It is possible to distinguish:

- so-called virtualization „PC in PC” constituting emulation of equipment stores of the host with using the software started in specific

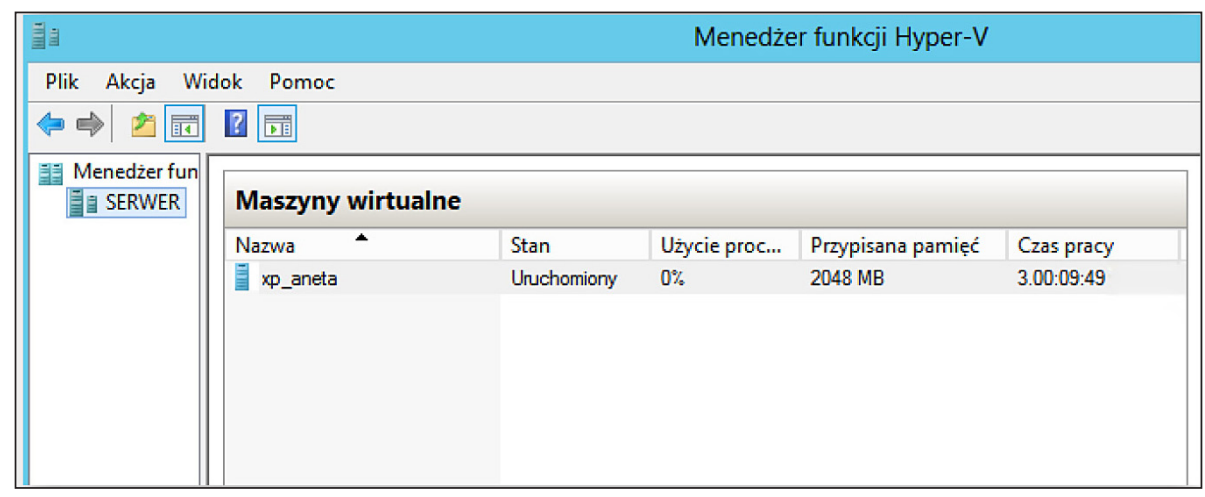

Fig. 3. Virtual machine application Hyper-V 


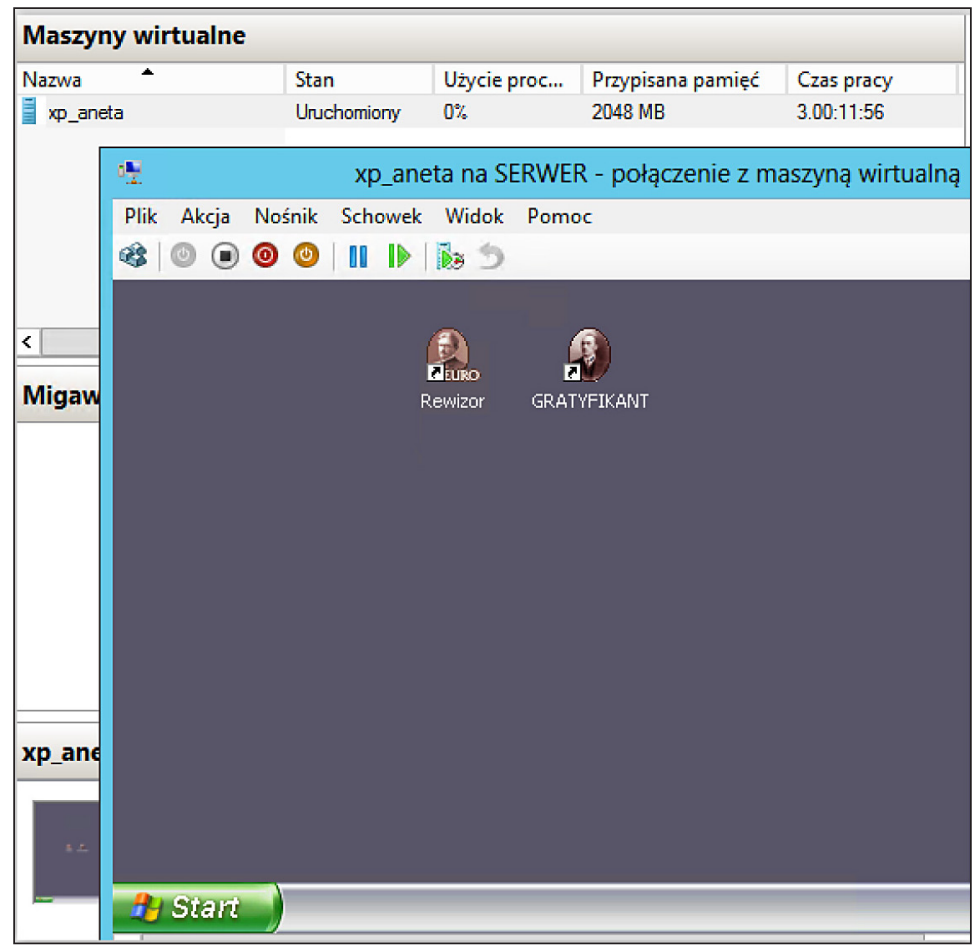

Fig. 4. System of the guest in the application Hyper-V

operating systems - very useful solution in testing purposes of the new software and the verification of saving viruses in terms of the presence;

- virtualization of application, that is making the application server available by hipervisor to the specific workstation and at using the desktop installed on operating systems of the software using it on the PC, what allows for the fast collective update or her blockade;

- client virtualization, which is the separation of the physical location of the server located on the hardware resources that reside on them, and client virtual machine images, from the place where the user is located and the use of so-called. thin clients - in the cur- rent version outdated computers with weak resources connecting to the virtual desktop infrastructure by local - LAN or wide WAN network with TCP / IP protocol with high throughput.

The most economically viable form of virtualization for the purpose of ERP applications running under DOS client virtualization, which from the server to the data repositories containing client virtual machines available on request to users for efficient use of existing resources, and often outdated information. therefore economically justified because the producer is providing with the version core of hypervisor Hyper-V system completely free of charge. It is fully functional platform of virtual machines.

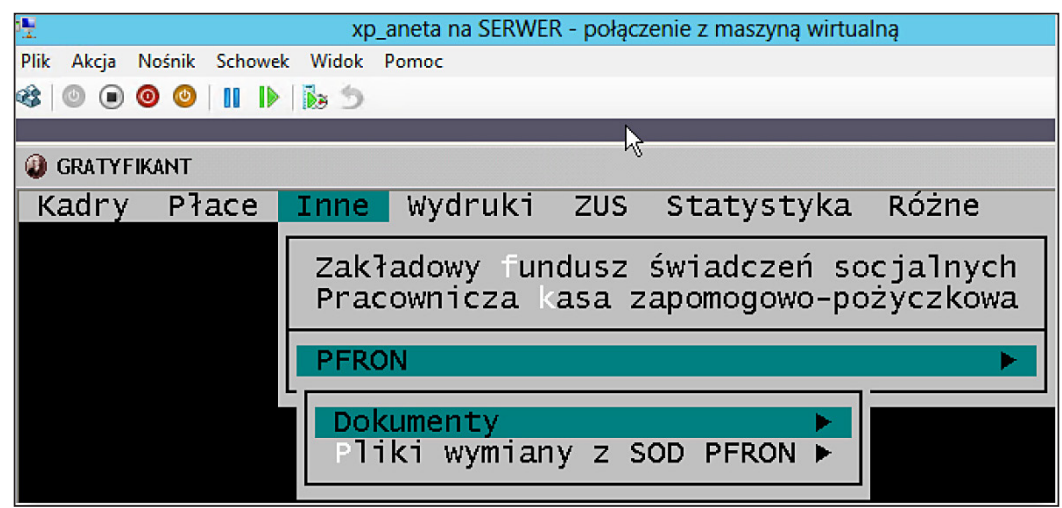

Fig. 5. Demonstration application under the system audit DOS 


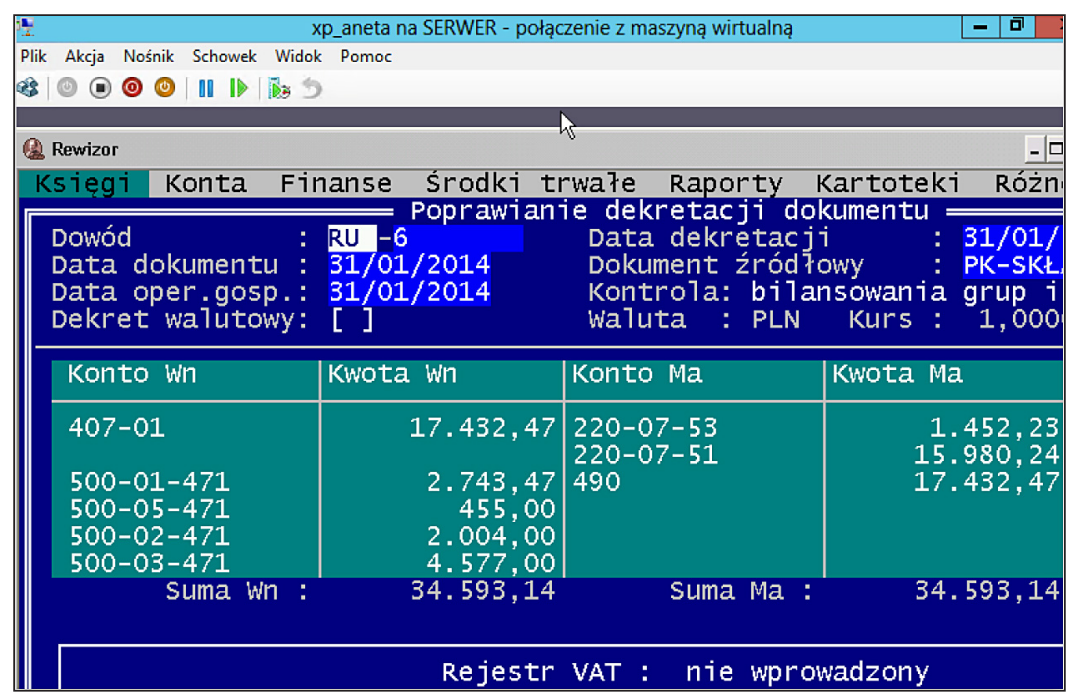

Fig. 6. Accounting application working under the DOS system audit without the graphic interface GUI

As a Windows PowerShell shell is enclosing with course books developed tools also free of charge enabling in the graphic way to create virtual machines and managing existing resources stayed. This system has both a Firewall and Defendera which are responsible for a safety.

However of scripts of such an application can be a few. Their choice is affecting the ergonomics from the side of the user or the administrator, the speed and the stability of action. As a result of run examinations I will focus on most optimum in terms of the productivity and the stability termination streamlining running a business in the current form.

The discussed solution is based on hipervisor in the version core. On account of as a rule very much big burdening the server with virtual machines isn't shown applying the graphical environment. Fully a management and a resource allocation of the host enable the operator Hypervisor. Existing tools are monitoring using resources by every customer. From here exceptionally effectively it is possible to exploit the entire power of the server and if some of systems of guests is turned off, to redirect dismissed stores to other virtual machine. The most he is an optimal system of the guest available for a dozen or so years Windows XP in the version 32-bit. His versatility consists in the service very much of many ERP applications working under control DOS and the possibility of configurations of parameters required by ERP. available tools of integration effectively are emulating action of the keyboard. Merging with such a virtual machine with the help available already from the system windows to get
98 client applications supporting the protocol of the RDP communication it is possible redirecting the customer of interrupts being responsible for parallel ports to the system and terrace. The printout which is being carried out on the machine of the host is staying in their result redirected to the printer connected in terms of physics to the port of the customer. To be redirected perhaps "move" both on parallel ports, privates as well as USB. using the redirection ,of move" on a serial port to get it is possible fiscal printout at the ward with the help of the server being in a head office. Also bar code reader connected by a serial port, USB or keyboard to the computer of the guest will actually be active in the branch distant from the head office of the company.

On account of very long supporting by the producer the system windows XP his licence on the current computer it is possible virtualize and as operating systems of the so-called thin customer to use distribution free of charge of the system Linux. It will only be used to start the application communicating with hypervisor with the help of the RDP protocol. Many available tools exist free of charge as additional applications downloaded from appropriate repositories. A functionality of display layer which must provide the simultaneous access for users for everyone to many desktops of virtual machines is an important difference of such a solution simultaneously, in the opposite to layer visualization used at virtualization of very servers, where only an administrator is gaining access to the desktop of the system, whereas the user is using only services made available on the server. 


\section{CONCLUSIONS}

Presented answers virtualization in the different form are ensuring benefits in the IT organization. many of mentioned above technologies connected mainly with the emulation are known and used in systems of the ERP class, some whereas, so like full virtualization of servers and desktops, are relatively new in terms of the realization in the business. virtualization to treat belongs not as the way to minimize the number of physical devices in the infrastructure of the business organization, but as the full support system delivering services backing the ERP software up under DOS in the effective way. Planning virtualization one should however choose stores of the IT infrastructure of the organization most appropriate in the current situation solution and to develop them in line with the earlier developed strategy virtualization. Such an approach will help to maximize swimming benefits from this technology.

\section{REFERENCES}

1. Popek G.J., Goldberg R.P.: Formal requirements for virtualizable third generation architectures. Communications of the ACM, Vol. 17 (7), 1974.

2. Promotional Materials Microsoft Corporation, VMware Inc., NetApp Corporatio. 